\title{
Mucosal Vaccine Delivery Using Mucoadhesive Polymer Particulate Systems
}

\author{
Chong-Su Cho ${ }^{1}$ Soo-Kyung Hwang ${ }^{1,2} \cdot$ Min-Jeong Gu${ }^{1} \cdot$ Cheol-Gyun Kim ${ }^{1}$ • \\ Seo-Kyung Kim ${ }^{1}$ Do-Bin Ju ${ }^{1}$ Cheol-Heui Yun ${ }^{1,3,4} \cdot$ Hyun-Joong Kim $^{2}$ (D)
}

Received: 23 May 2021/Revised: 24 June 2021/Accepted: 25 June 2021/Published online: 25 July 2021

(C) The Korean Tissue Engineering and Regenerative Medicine Society 2021

\begin{abstract}
Vaccination has been recently attracted as one of the most successful medical treatments of the prevalence of many infectious diseases. Mucosal vaccination has been interested in many researchers because mucosal immune responses play part in the first line of defense against pathogens. However, mucosal vaccination should find out an efficient antigen delivery system because the antigen should be protected from degradation and clearance, it should be targeted to mucosal sites, and it should stimulate mucosal and systemic immunity. Accordingly, mucoadhesive polymeric particles among the polymeric particles have gained much attention because they can protect the antigen from degradation, prolong the residence time of the antigen at the target site, and control the release of the loaded vaccine, and results in induction of mucosal and systemic immune responses. In this review, we discuss advances in the development of several kinds of mucoadhesive polymeric particles for mucosal vaccine delivery.
\end{abstract}

Keywords Polymeric particles $\cdot$ Mucosal vaccination $\cdot$ Antigen delivery $\cdot$ Mucoadhesive polymers

Chong-Su Cho and Soo-Kyung Hwang equally contributed to this work.

Cheol-Heui Yun

cyun@snu.ac.kr

$\triangle$ Hyun-Joong Kim

hjokim@snu.ac.kr

1 Department of Agricultural Biotechnology and Research Institute of Agriculture and Life Sciences, Seoul National University, 1 Gwanak-ro, Gwanak-gu, Seoul 08826, Republic of Korea

2 Lab. of Adhesion \& Bio-Composites, Department of Agriculture, Forestry and Bioresources, Seoul National University, 1 Gwanak-ro, Gwanak-gu, Seoul 08826, Republic of Korea

3 Institute of Green-Bio Science and Technology, Seoul National University, Pyeongchang-gun, Gangwon-do 25354, Seoul, Republic of Korea

4 Center for Food and Bioconvergence, Seoul National University, 1 Gwanak-ro, Gwanak-gu, Seoul 08826, Republic of Korea

\section{Introduction}

Vaccination has recently become more important as one of the most successful medical treatments due to the improvement of world health and reduction of the prevalence of many infectious diseases [1-3] including Coronavirus disease-19 (COVID-19) first reported in late 2019, it has become a pandemic across the world with about 150 million infections and about 3 million deaths as of May in 2021. The vaccination is aimed to induce and harness protective effector and memory immunity by comprising neutralization of antibodies together with cytotoxic and helper $\mathrm{T}$ cells [4].

Recently, the development of the vaccine is focused on subunit vaccines, such as protein, toxoid, peptide, or DNA, generally regarded as less reactogenic and less risk of reversion than live-attenuated or killed whole-pathogens although this is challenging for the finding of sufficient immunogenicity due to the most depletion of the innate immune stimulus of the subunit ones [5]. Over the last decades, vaccine technology has allowed us to find out the 
administration route because parenteral vaccination is not enough for the induction of immunity at the site of pathogen entry such as mucosal surfaces although most of the licensed subunit vaccines are administered parenterally. It is now well accepted that mucosal vaccination induces effective humoral and cellular immunity than parenteral one because mucosal immune responses are the first line of defense against most pathogens and protection of mucosal immunity [5]. For the development of successful mucosal vaccines, several immunological and physiological aspects should be taken into account [5]. Firstly, the mucosal vaccines must be resistant to site-specific $\mathrm{pH}$ and stable in an enzymatic environment, secondly, they should be delivered to mucus and epithelium sites, thirdly, they should be adapted to interactions with mucus, fourthly, they should be captured by appropriate antigen-presenting cells (APCs), lastly, they should overcome tolerogenic nature of the mucosa. Also, compliance of the patients should be considered for vaccination because a large number of people in countries where endemic infections are present should be vaccinated without non-invasiveness, pain, and trained medical staff for administration [1]. Therefore, there is an increasing demand for mucosal vaccination. As an alternative to parenteral one, there are several advantages of mucosal vaccination, such as easy administration, allowing mass vaccination, needle-free, simple production, and lower costs [1]. Until now, only a few mucosal applications of the subunit vaccine formulations are successful [4-6] because of the limited efficient delivery systems.

Polymeric systems such as polymeric nano-/microparticles for mucosal vaccine delivery are very important because they provide delivering vaccines to a specific target site, they control the release of vaccines in the mucosal sites, they protect vaccines from harsh gastric $\mathrm{pH}$, digestive enzymes and bile juices in the gastrointestinal (GI) tract, and easy modifications of polymeric systems to meet physicochemical properties. Also, the polymeric nano-/ microcarriers can enhance the immune responses to mucosally delivered vaccines because they have greater access to immune compartments in Peyer's patches compared to soluble vaccines themselves. Furthermore, they pave their way via the paracellular route for entering underlying lymphoid cells.

Limitation of mucosal vaccine delivery is the rapid clearance of administered vaccines. The vaccines should pass from the mucosal barrier to mucosal lymphoid tissues to reach the site of action. Therefore, mucoadhesive polymeric particulate systems are very important because they prolong the residence of the vaccines at the mucosal sites and they can enhance the immune responses to mucosally delivered vaccines.
Selection of drug delivery route is very critical for achieving therapeutic success and enhancing bioavailability in medical science. Generally, there are two kinds of delivery route such as parenteral and non-parenteral routes. Various parenteral routes, like intravenous, intramuscular, subcutaneous, and intradermal routes have been used due to the high bioavailability although they have several limitations such as invasiveness, painful to patients and necessity for trained personal. As alternate routes, several non-parenteral routes such as mucosal and topical routes have been recently attracted to solve the limitations of the parenteral one.

In this review, we cover recent advances in the development of mucoadhesive polymeric particles including chitosan, cellulose, Eudragit and hybrid for mucosal vaccination. The schematic illustration of the review contents is shown in Fig. 1.

\section{Mucosal immunity}

Mucosal tissues keep a fine equilibrium with the microbiota and regulate the induction of tolerance against dietary antigens while immunological activity against many pathogens [7-9]. The mucosal immune system consists of an integrated tissue network, lymphoid and constitutive cells, and effector molecules such as cytokines, chemokines, and antibodies [10]. These several factors respond to pathogens or vaccines via an orchestra of cellular processes for innate and adaptive immune responses as shown in Fig. 2 [11].

Antigen-specific immune responses are induced in the organized mucosa-associated lymphoid tissues (MALT) as gut-associated lymphoid tissues (GALT) or Peyer's patches in the intestinal mucosa [11]. The MALT functions independently from the systemic immune response and it consists of APCs such as dendritic cells (DCs) and T lymphocytes, and plasma cells such as B lymphocytes [11]. Antigen-specific antibodies as secretory $\operatorname{IgA}$ (sIgA) made by the plasma cells are secreted to the mucosal surface to bind, neutralize and eliminate the pathogens. The induction of $\operatorname{sigA}$ response is very important for successful mucosal vaccination as it is the first line of defense against pathogens [11].

The microfold $(\mathrm{M})$ cells as specialized epithelial cells and specifically located on the follicle-associated epithelium (FAE) in the mucosal tissues are the most targets for vaccine delivery via the mucosal administration for induction of mucosal immunity because they capture and transport pathogens or vaccines across the epithelial barrier to lymphoid cells [7]. The transposed vaccines are then passed to B cells for activation of mucosal response via the 
Fig. 1 Schematic illustration of the contents



secretion of $\operatorname{sIg} \mathrm{A}$ or to DCs for activation of humoral response via the initiation of $\operatorname{IgA}$ production.

\section{Characteristics of mucosal vaccine delivery by polymeric systems}

The use of polymeric systems for mucosal vaccine delivery has been attracted as they provide an advantage of delivering vaccines to a specific target site, they control the release of vaccines from their grip in the mucosal sites, they can protect vaccines from harsh gastric $\mathrm{pH}$, digestive enzymes and bile juices in the gastrointestinal (GI) tract, and easy modifications of polymeric systems to tune up physicochemical properties such as particulate, surface charge, particle size, and solubility can be available for the efficacy of mucosal vaccine delivery [11]. Especially, particulate of the polymer can enhance the immune responses to mucosal delivered vaccines because particulate vaccines have greater access to immune compartments in the MALT through M cells in Peyer's patches compared to soluble vaccines $[12,14]$. Also, particulate vaccines find their way via the paracellular route for entering underlying lymphoid cells from the follicle-associated epithelium (FAE) in the mucosal tissues [13].

\section{Different mucosal vaccination route}

Various parenteral routes such as intravenous, subcutaneous, and intramuscular hold the whole stake of current drug delivery. However, mucosal administration has been recently attracted as an alternate route due to the invasiveness of parenteral ones. In this section, several mucosal antigen deliveries by different routes such as oral, nasal, rectal vaginal, and ocular will be discussed. And the specification of anatomical and physiological characteristics of the mucosal sites for the selection of suitable routes is summarized in Table 1 [15]. Furthermore, advantages/ disadvantages of each vaccination route are summarized in Table 2.

\subsection{Oral Route}

The oral route is the easiest administration of the vaccine. Generally, the higher concentration of the vaccine may be necessary for its efficacy as on passage via the GI tract as the concentration gets diluted [16]. The advantages of oral vaccination are safe, painless, low-cost, and not required a trained person for administration. There are two kinds of ways to pass through the GI tract. One is to swallow the vaccine whereas the other is to enter the oral cavity.

The major disadvantage of the oral route administration requires a higher concentration of the vaccine for better effectiveness [17] although polymeric nanoparticles (NPs) were used for oral DNA vaccines [18]. Currently licensed oral vaccines for human use cholera, poliomyelitis, rotavirus, and typhoid [19]. Among oral route, the sublingual route has recently attracted because it improves the patient's compliance, reduce the time for the onset of drug action, and increases the bioavailability although a small number of drugs for angina pectoris, hypertensive crises, breakthrough cancer pain, and migraine are commercially available because eating, drinking, or smoking can affect drug adsorption and extended-drug release cannot be obtained. Also, buccal route as an another oral route has several advantages such as very quick absorbed medication, not-going of drugs through the digestive system, and non-necessity of swallowing drugs although the disadvantages of the buccal route is almost similar with the sublingual one.

\subsection{Nasal route}

The nasal vaccination is one of the popular alternative vaccination methods as it is easily accessible for self- 




Fig. 2 Schematic diagram of various immune responses induced by particulate vaccine system. Upon encounter with an antigen, B cells convert themselves to antibody secreting plasma cells that produce antibodies for excreting the pathogens to mucosal surfaces (mucosal response) whereas dendritic cells (DCs) present the antigen via major histocompatibility complex (MHC) class I and class II molecules to $\mathrm{CD} 8+$ and CD4 + T-cells. Activation pathway of CD8 + T cells and CD4 + Th1 cells produces cytotoxicT lymphocytes (CTL) and

administration, the nasal cavity is highly vascularized with a large surface area for antigen uptake and a relatively small dose is required due to the direct delivery of vaccines to the targeted site although most antigens have less affinity for the nasal epithelium with a fast clearance rate [19]. On the other hand, there are several disadvantages such as immediate removal of free antigen from the nasal passage, activated macrophages that kill intracellular pathogens or infected cells (cellular response) while activation pathway of CD4 + Th2 cells produce sactivated B lymphocytes that secrete antibodies for neutralization of extracellular pathogens (humoral response). Adapted from Singh et al., Chitosan-based particulate systems for the delivery of mucosal vaccines against infectious diseases. International Journal of Biological Macromolecules 2018, 110, 54-64, with permission of Elsevier [11]

very poor absorption of the nasal epithelial layer, and comparatively low immune response [16]. Only two licensed intranasal vaccines for use in humans are influenza and diphtheria [16]. Among nasal route, vaccine delivery via pulmonary route has gained increasing attraction because this route omits the use of needles, can elicit immunity at the site of entry for many pathogens that can 
Table 1 The specification of anatomical and physiological characteristics of mucosal sites (modified from Ref. [16])

\begin{tabular}{|c|c|c|c|c|c|c|}
\hline \multirow{2}{*}{$\begin{array}{l}\text { Sites } \\
\text { parameters }\end{array}$} & \multicolumn{2}{|l|}{ Oral } & \multirow[t]{2}{*}{ Nasal } & \multirow[t]{2}{*}{ Rectal } & \multirow[t]{2}{*}{ Rectal } & \multirow[t]{2}{*}{ Ocular } \\
\hline & Buccal & Sublingual & & & & \\
\hline Mucosal thickness & $500 \sim 800 \mu \mathrm{m}$ & $100 \sim 200 \mu \mathrm{m}$ & $700 \sim 1000 \mu \mathrm{m}$ & $1 \mathrm{~mm}$ & $200 \sim 300 \mu \mathrm{m}$ & $\begin{array}{l}520 \mu \mathrm{m} \\
\text { For corneal and } 52.56 \pm 19.02 \\
\mu \mathrm{m} \\
\text { For conjunctival }\end{array}$ \\
\hline Surface area & $200 \mathrm{~m}^{2}$ & & $160 \mathrm{~cm}^{2}$ & $300 \mathrm{~cm}^{2}$ & $70 \mathrm{~cm}^{2}$ & $2 \mathrm{~cm}^{2}$ \\
\hline $\mathrm{pH}$ & $6.2 \sim 7.4$ & & $5.0 \sim 6.5$ & $7.8 \sim 8$ & $4 \sim 5$ & 7 \\
\hline $\begin{array}{l}\text { Biological } \\
\text { secretions }\end{array}$ & $0.5 \sim 2 \mathrm{~L} /$ day & & $1 \sim 1.5 \mathrm{~L} /$ day & $\begin{array}{l}1800 \sim \\
1825 \\
\mathrm{ml} / \text { day }\end{array}$ & $4 \sim 5 \mathrm{ml} /$ day & $1.2 \mu 1 / \mathrm{min}$ \\
\hline
\end{tabular}

Table 2 Summary of advantages/disadvantages of each vaccination route

\begin{tabular}{|c|c|c|c|}
\hline Route & Advantages & Disadvantages & Ref. \\
\hline Oral & $\begin{array}{l}\text { Safe, painless, low-cost, and not necessary of a trained person } \\
\text { for administration }\end{array}$ & Require a higher concentration of used vaccine & [18] \\
\hline Nasal & $\begin{array}{l}\text { Easy access for self-administration, highly vascularized of nasal } \\
\text { cavity, requirement of relatively small dose of vaccine }\end{array}$ & $\begin{array}{l}\text { Immediate removal of vaccine from nasal passage, poor } \\
\text { absorption of nasal epithelial layer, low immune response }\end{array}$ & [17] \\
\hline Rectal & $\begin{array}{l}\text { Strong induction of immune response, generation of genital and } \\
\text { rectal tract immunity }\end{array}$ & Poor acceptability & [23] \\
\hline Vaginal & $\begin{array}{l}\text { Generation of strong immune response in female reproductive } \\
\text { system }\end{array}$ & Providing not-sufficient hard immunity & [25] \\
\hline Ocular & Very effective in herpes simplex virus- 1 & $\begin{array}{l}\text { Very difficult to penetrate drugs at posterior segment and } \\
\text { anterior one of the eye }\end{array}$ & [27] \\
\hline
\end{tabular}

cause pulmonary diseases, and there is a very large surface area available for interaction with the used vaccines. However, pulmonary delivery device should be designed for avoiding patient contamination and transmission of diseases. Also, due to limited knowledge of pulmonary immunology,

not many clinical trials were performed by far.

\subsection{Rectal route}

Rectal vaccination has been used to prevent several diseases such as enteric pathogens, cancer, and sexually transmitted diseases [20]. Generally, the immune response is more strongly induced at the vaccination site and it is possible to generate both genital and rectal tract immunity via the rectal vaccination [21]. However, this rectal vaccination is not widely used due to poor acceptability [22].

\subsection{Vaginal route}

Vaginal vaccination has been applied for the prevention of pathogens transmitted sexually via the genital tract, such as
HIV, HPV, and chlamydia [19]. This is one of the challenging methods to generate an immune response because immunological features of the female reproductive system dramatically change according to hormonal fluctuations during the menstrual cycle [23]. However, this vaccination has not been explored as extensively because it can immunize only females and it does not provide sufficient herd immunity [24].

\subsection{Ocular route}

Ocular vaccination has been applied for the prevention of ocular pathogens that cause corneal scarring and blindness [25]. In particular, herpes simplex virus (HSV)-1 is a major cause of infectious blindness worldwide although traditional immunization approaches using live, attenuated, or inactivated vaccines, or conventional antigens injected intramuscularly or parenterally are ineffective for achieving ocular immunization [25]. Ocular surfaces are a significant portal of entry for many pathogens. The lymphoid components in the ocular mucosa are composed of the MALT. The local immune system to serve the ocular 
surface is the mucosal barrier for protecting the eye [25]. However, it is very difficult to penetrate drugs at the posterior segment and anterior one of the eye although microneedle-mediated vaccine delivery-based research has gained great interest in recent years [26].

\section{Mucoadhesive polymeric particulate system}

A major challenge of mucosal vaccine delivery is the rapid clearance of administered vaccines due to the brief contact with mucus lay. The vaccines have to pass from the mucosal barrier to mucosal lymphoid tissues to reach the site of action [30]. Therefore, prolonging the residence of the vaccines at the mucosal membranes is important for providing maximum immune effect. Also, polymeric particles can enhance the immune responses to mucosally delivered vaccines. Furthermore, particulate vaccines have greater access to immune compartments in MALT compared to soluble vaccines [12]. In this section, we will discuss several mucoadhesive polymeric particulate systems for the effective induction of mucosal immune responses.

\subsection{Chitosan-based mucoadhesive system}

The chitosan obtained from chitin as the $N$-deacetylated derivative has been applied for biomedical applications such as wound dressing [31], contact lenses [32], artificial skin [32],

and drug delivery carrier [33], due to the excellent biocompatibility, bioavailability low toxicity, and adhesive capabilities [30]. Among these applications, chitosan has been much applied for drug delivery because of mucoadhesive ability and control of drug release even in its unmodified form and easy preparation of chitosan-based particles by physical and chemical methods as explained in the 5.1.1 section. However, chitosan in its unmodified form has limitations such as high solubility at acidic $\mathrm{pH}$, rapid clearance from the body [34]. Therefore, the more mucoadhesive property should be desirable for the mucosal vaccine delivery since the administered vaccines must successfully interact with mucosal membranes to remain at the mucosal site for a longer time for providing maximum vaccine effect by using several kinds of thiolated chitosan derivatives as shown in Fig. 3 [30].

Many researchers have developed chitosan derivatives as the second-generation mucoadhesive chitosan. In this section, we will discuss methods of chitosan particles and several mucoadhesive chitosan derivatives for mucosal vaccine delivery.

\subsubsection{Preparation of chitosan-based particles}

Chitosan-based particles for vaccine delivery can be prepared by physical or chemical methods although each method has advantages and disadvantages. Generally, physical methods are preferred to the chemical ones because the vaccines chemically modified by crosslinking agents are degraded by organic solvents used for a chemical reaction [11]. Several methods for the preparation of particles are discussed.

5.1.1.1 Physical method Chitosan-based particles can be prepared by ionic crosslinking between cationic chitosan derivatives and anionic low molecular weight compounds such as sodium sulfate or tripolyphosphate (TPP) via spontaneous ionotropic gelation to microparticles or nanoparticles according to the molecular weight of chitosan and concentration of anionic compounds. The advantages of the ionic crosslinking method are spontaneously forming nanoparticles upon adding aqueous TPP solution incorporated with vaccines to the chitosan aqueous solution and the loaded vaccines cannot be degraded by chemical crosslinkers, organic solvents, and high temperature [27]. Another physical method is precipitation or complex coacervation. This method is to form chitosanbased microparticles and nanoparticles depending on the molecular weights of used chitosan and isoelectric point of vaccines. The vaccines can be abundantly loaded within the chitosan-based particles by physical adsorption on the particles and ionic interaction between cationic chitosanbased particles and anionic vaccines. The disadvantage of the precipitation method is no release of the vaccines from the loaded vaccines due to the strong ionic interaction between cationic chitosan and anionic vaccine.

5.1.1.2 Chemical method Chitosan-based particles are prepared via a chemical interaction between the amino groups of chitosan and cross-linking agents such as glutaraldehyde, $p$-phthaldehyde, ascorbyl palmitate, and dehydroascorbic palmitate [28]. The chemical cross-linking takes place either one or two steps. The first step is to form a water/oil emulsion in which chitosan and vaccine are in the water phase emulsified into the external immiscible solvent. The second step is gradually to add the crosslinking agent and finally, the prepared particles are separated. Some additives may be used to increase the stability and loading efficiency of the used vaccines [29].

\subsubsection{Thiolated chitosan derivatives}

Several thiolated chitosan derivatives as shown in Fig. 3 [30] were prepared for vaccine delivery. These thiolated chitosan derivatives may cause thiol/disulfide exchange 
<smiles>COC1C(CO)OC(C)C(NC(=O)C(N)CS)C1O</smiles>

Chitosan-cysteine<smiles>COC1C(CO)OC(C)C(NC(=[NH2+])CCCS)C1O</smiles>

Chitosan-4-thio-butylamidine<smiles>COC1C(CO)OC(C)C(NC(=O)CS)C1O</smiles>

Chitosan-thioglycolic acid<smiles></smiles><smiles>COC1C(CO)OC(C)C(O)C1NC(=O)c1ccc(S)cc1</smiles><smiles>COC1C(CO)OC(C)C(NC(=O)C(CS)NC(C)=O)C1O</smiles>

\section{Chitosan-glutathione}



Chitosan-6-mercaptonicotinic acid

Chitosan- $N$-acetylcysteine<smiles>COC1CC(CO)OC(C)C1NC(=O)c1ccc(S)cc1</smiles><smiles>COC1C(CO)OC(C)C(NC(=O)C(NC(C)=O)C(C)(C)S)C1O</smiles>

Chitosan-4-mercaptobenzoic acid

Chitosan- $N$-acetylpenicillamine

Fig. 3 Chemical structures of the presented chemical-modified chitosan variants. Adapted from Islam et al., Mucoadhesive Chitosan Derivatives as Novel Drug Carriers, Current Pharmaceutical Design, 2015, 21, 4285-4309 with permission of Bentham Science [30] 
reactions with mucus by leading to disulfide bond formation between the thiolated chitosan and mucus layer and result in mucoadhesion [35] although the exact mechanism on the mucoadhesion between them remains unclear.

Verheul et al. prepared covalently stabilized polymeric nanoparticles between thiolated trimethyl chitosan (TMC) and thiolated hyaluronic acid (HA) via ionic gelation followed by disulfide formation to load ovalbumin (OVA) [36] because the polyelectrolyte complex nanoparticles formed by TMC and HA is limited in physiological condition. The results indicated that OVA-loaded TMC-s-sHA nanoparticles showed higher IgG titers after nasal administration in mice than OVA-loaded TMC/HA ones due to the stabilization of the TMC-s-s-HA nanoparticles although they did not check immune responses by the mucoadhesive property of the stabilized nanoparticles.

Sinani et al. prepared aminated plus thiolated chitosan nanoparticles by ionotropic gelation method with TPP to enhance mucoadhesion and adjuvanticity of the vaccine [37]. The mucoadhesion results obtained by the work of adhesion and peak detachment force were the highest with amination plus thiolation of chitosan compared to the results of chitosan and aminated chitosan due to strengthening the mucoadhesive bonds with sialic acid groups of mucin chains, and covalent bonding to mucus glycoproteins [38]. Also, high levels of systemic antibodies such as $\mathrm{IgG}, \operatorname{IgG} 1$, and $\operatorname{IgG} 2 \mathrm{a}$ and mucosal $\operatorname{sg} \mathrm{A}$ in vaginal washes were successfully appeared after nasal vaccination in mice using bovine serum albumin (BSA) as a model vaccine. Furthermore, a mixed Th1/Th2 immune response was observed, suggesting great potential for nasal application of vaccines.

\subsubsection{Quaternized chitosan derivatives}

Many researchers have studied chitosan as a drug carrier due to its good biocompatibility and biodegradability. However, the low water solubility of the chitosan limits the application of it as a permeation enhancer for the mucosal surfaces above the $\mathrm{pH}$ of $6 \sim 6.5$. As alternatives, quaternized chitosan derivatives have been used as vaccine carriers due to water solubility over a wide range of $\mathrm{pH}$, mucoadhesive property with a significant reduction of cytotoxicity, higher cell permeability, and stronger antigenbinding ability by more positive changes [39]. In this section, we discuss mucosal vaccination using quaternized chitosan derivatives.

Among quaternized chitosan derivatives, mostly there are two kinds of quaternized chitosan such as trimethyl chitosan (TMC) and hydroxypropyl trimethyl ammonium chloride chitosan (HACC).

Marasini et al. prepared a lipopeptide-based vaccine (LPV) against group A Streptococcus(GAS)/dextran/TMC nanoparticles by a double emulsion to check their ability to be taken up by DCs in vivo [40]. It was found that the LPV/ dextran/TMC showed improved uptake by DCs and induced DC maturation. Also, the combination of lipopeptide conjugated with Toll-like receptor agonist lipidic moiety and TMC-based nanoparticles showed the highest stimulation of humoral immune responses and systemic antibodies in sera after nasal immunization in mice due to the adjuvanting property of lipopeptide.

$\mathrm{Li}$ et al. prepared nanoparticles composed of pVAXI plasmid as an anticaries DNA vaccine and TMC by the mixed complex coacervation and ionotropic gelation technique to elicit mucosal and systemic immune responses [41]. The results indicated that higher specific $\operatorname{IgG}$ antibodies were obtained in rats immunized with pVAXIWapA/TMC nanoparticles compared with naked pVAXIWapA after nasal immunization. Also, Anti-WapA IgA and IgA antibody titers were significantly higher after nasal administration than intramuscular one or naked pVAXIWapA with fewer enamel, and dentin moderate lesions, a suggestion of a promising candidate for anticaries vaccine development.

Abkar et al. prepared nanoparticles composed of Brucella. B. melitensis Omp 31 as a subunit vaccine against brucellosis and TMC by ionic gelation to study immune response [42]. The results indicated that Omp31/TMC nanoparticles elicited a mixed T helper 1 (Th1) and Th17 immune response, and stimulated higher antigen-specific cell proliferative response with significant protection of pathogen infection after oral immunization in mice whereas Omp31 TMC nanoparticles induced Th1-Th2 immune responses after intraperitoneal immunization, suggesting that the administration route affects the type of immune response.

Nevagi et al. prepared nanovaccine composed of antigen peptide (having B-cell epitope and T-helper epitope)-conjugated $\alpha$-polyglutamic acid and TMC by a complex coacervation method to protect GAS pathogen as one of the top-ten human pathogens in terms of mortality [43]. This nanovaccine induced higher mucosal and systemic antibody titers compared with antigen with mucosal adjuvant cholera toxin B or antigen mixed with TMC. Also, a reduced bacterial burden was obtained in nasal shedding, throat swabs, and nasopharyngeal-associated lymphoid tissue (NALT) of mice after nasal challenge with the MIGAS strain, a suggestion of conjugation of peptide antigen to the anionic polymer as a promising strategy for vaccine delivery. Similarly, they prepared another nanovaccine composed of antigen peptide (having B-cell epitope of $\mathrm{J} 8$ and T-helper epitope of $\mathrm{P} 25$ )-conjugated polyglutamic acid and TMC by a complex coacervation method to protect GAS pathogen [44]. The nanovaccine prepared from a peptide conjugated with 10 residues of 
polyglutamic acid and fungal TMC induced the highest systemic antibody titers and produced antibodies that were opsonic against GAS pathogens after nasal immunization in mice, an indication that proper anionic residue numbers and source of TMC are crucial in inducing an efficient immune response.

Recently, Jearanaiwitayakul et al. prepared nanovaccine composed of non-structural protein (NS1) of dengue virus (PENV) vaccine and TMC by the ionic gelation method to protect dengue virus as the most common mosquito-borne viral disease [45]. The nanovaccine potentially stimulated monocyte-derived DCs (MoDCs) and resulted in increased expression of CD83 as the maturation marker, and CD80 and CD86 as costimulating molecules, and marked secretion of innate immune cytokines. Also, this nanovaccine strongly elicited both antibody and $\mathrm{T}$ cell responses with higher production of $\operatorname{IgG}, \operatorname{IgG} 1, \operatorname{IgG} 2 \mathrm{a}$, and activated $\mathrm{CD}^{+} \mathrm{T}$ cells after intraperitoneal immunization in mice although they did not compare with immune responses between systemic immunization and mucosal one.

Zhao et al. prepared nanovaccine composed of New castle disease (ND) vaccine and $N$-2-hydroxypropyl trimethyl ammonium chloride chitosan (N-2-HACC) by the ionic gelation method to protect $\mathrm{ND}$ as a serious viral disease of poultry [46]. The nanovaccine showed no damage to the ND vaccine after loading into nanoparticles with low cytotoxicity. Also, it showed much stronger cellular, humoral, and mucosal immune responses than commercial attenuated love ND vaccine after immunization in chickens, a suggestion of a potential vaccine carrier of N-2HACC. Also, they prepared nanovaccine composed of ND vaccine and N-2-HACC by the complex coacervation method to protect ND in chickens [47]. The nanovaccine showed higher stability with lower cytotoxicity and sustained release of the vaccine from the nanoparticles after an initial burst release. Also, it induced higher titers of $\mathrm{IgG}$ and IgA antibodies, promoted proliferation of lymphocytes, and showed higher levels of interleukine-2 (IL-2), IL-4, and interferon- $\gamma($ IFN- $\gamma$ ) than the commercially combined attenuated live vaccine after nasal immunization in chickens, a suggestion of immense application potential in the poultry farm. Furthermore, they prepared nanovaccine composed of ND virus DNA vaccine with C3d6 adjuvant and N-2-HACC INO-carboxymethyl chitosan (CMC) nanoparticles by the complex coacervation to protect ND in chickens [48]. It was found that the DNA vaccines were sustainably released from the nanovaccines after an initial burst release. Also, the nanovaccines produced not only higher anti-ND-vaccine $\operatorname{IgG}$ and $\operatorname{sg} \mathrm{A}$ antibodies but also stimulated lymphocyte proliferation with triggering higher IL-2, IL-4, and IFN- $\gamma$ levels after nasal immunization in chickens than infra muscular one, suggesting that mucosal route is better than systemic one for better immune responses.

Glycol chitosan (GC) prepared by conjugation with ethylene glycol branches to chitosan increases water solubility at an acidic/neutral $\mathrm{pH}$ values, provides steric stabilization, and shows mucoadhesive property compared with chitosan itself [49].

Powar et al. prepared nanovaccine composed of hepatitis B surface antigen (HBsAg) and GC by the complex coacervation method to protect hepatitis B virus [50]. The nanovaccine showed a lower nasal clearance rate in the nasal cavity and better mucosal uptake compared to chitosan nanoparticles due to the attribution to the better mucoadhesion. Also, the nanovaccine induced higher antiHBsAg titers at salivary, nasal, and vaginal secretion sites than chitosan/HBsAg after nasal immunization whereas alum-based HBsAg vaccine injected subcutaneously as a positive control induced strong humoral but negligible mucosal immunity, suggestion of enhanced mucosal and systemic immune responses.

A selection of studies within the last 5 years on the application of chitosan derivative-based mucoadhesive particles for mucosal vaccine delivery is summarized in Table 3 .

\subsection{Cellulose derivative-based mucoadhesive particles}

Cellulose known as a highly abundant natural biopolymer composed of the main structural material of plant cell walls is a linear homopolymer of beta-(1-4)-linked 6 -glucopyranosyl units having a degree of polymerization of around $10,000 \sim 15,000$. Different cellulose molecules interact to form a large aggregate structure held together by mainly intermolecular hydrogen bonds between hydroxyl groups and result in insolubility in water and most organic solvent due to the high crystallinity. On the other hand, watersoluble cellulose derivatives, such as carboxymethyl cellulose (CMC), hydroxyethylcellulose (HEC), hydroxypropyl cellulose (HPC), quaternized cellulose (QC), and hydroxypropylmethylcellulose (HPMC) have been used in medical and pharmaceutical applications. In this section, we discuss mucosal vaccine delivery using cellulose derivative-based mucoadhesive particles.

Dennelly et al. prepared lyophilized solid dosage forms (LSDFs) composed of HIV-1 clade-C trimeric envelope glycoprotein vaccine (CN54gp140) and CMC to protect against HIV infection fuelled predominantly by heterosexual transmission [51]. The LSDFs showed prolonging vaccine stability compared to an aqueous-based vaccine and enhanced vaginal retention in a woman compared to conventional vaginal gel formulations due to the mucoadhesive property of the CMC. Also, the LSDFs boosted 
Table 3 Summary of Studies Using Chitosan Derivative-based Mucoadhesive Particles for Mucosal Vaccine Delivery

\begin{tabular}{|c|c|c|c|c|c|}
\hline Chitosan type & Antigen & Nanoparticle method & $\begin{array}{l}\text { Administration } \\
\text { route }\end{array}$ & Recipient & Ref. \\
\hline Thiolated MTC & OVA & Ionic gelation & Nasal & Mice & [45] \\
\hline $\begin{array}{l}\text { Aminoated and thiolated } \\
\text { chitosan }\end{array}$ & BSA & Ionic gelation & Nasal & Mice & [46] \\
\hline TMC & LPV & Double emulsion & Nasal & Mice & [49] \\
\hline TMC & pVAX-1-WapA & $\begin{array}{l}\text { Ionic gelation/complex } \\
\text { coacervation }\end{array}$ & Nasal & Rat & {$[50]$} \\
\hline TMC & Brucella B. melitensis Omp 31 & $\begin{array}{l}\text { Ionic gelation/complex } \\
\text { coacervation }\end{array}$ & Oral & Mice & [51] \\
\hline TMC & Antigen peptide vaccine & Complex coacervation & Nasal & Mice & [52] \\
\hline TMC & Antigen peptide vaccine & Complex coacervation & Nasal & Mice & [53] \\
\hline TMC & Dengue virus & Ionic gelation & Intraperitoneal & Mice & [54] \\
\hline HACC & Newcastle disease & Ionic gelation & Nasal & Chicken & [55] \\
\hline HACC & Newcastle disease & Complex coacervation & Nasal & Chicken & [56] \\
\hline HACC & $\begin{array}{l}\text { Newcastle disease with } \mathrm{C} 3 \mathrm{~d} 6 \\
\text { adjuvant }\end{array}$ & Complex coacervation & Nasal & Chicken & [56] \\
\hline GC & Hepatitis B surface & Complex coacervation & Nasal & Mice & [59] \\
\hline
\end{tabular}

Abbreviations: TMC trimethyl chitosan, BSA bovine serum albumin, $L P V$ lipopeptide-based vaccine, pVAX-1-WapA, plasmid VAX-1-wallassociated protein $\mathrm{A}$

systemic CN54gp140-specific antibody responses in subcutaneously primed mice after intravaginal immunization in the mouse model, indicating that the LSDFs are viable mucosal vaccine delivery system with promoting vaccine stability and facilitating intimate exposure of the used vaccine to the MALT of the female genital tract.

Singh et al. designed a $\mathrm{pH}-$ and mucoadhesive vaccine delivery carrier by thiolation of originally $\mathrm{pH}$-sensitive hydroxypropyl methylcellulose phthalate (HPMCP) used for enteric coating polymer by pharmaceutical industries as shown in Fig. 4 [52] to overcome several physical and biological barriers for oral vaccine delivery. They especially emphasized side-specific vaccine delivery in the small intestine before vaccine degradation in the stomach because the difficulty in delivering the vaccines via oral administration is owing to changes in $\mathrm{pH}$ values according to the different regions of the gastrointestinal (GI) tract; stomach ( $\mathrm{pH} 2.0 \sim 4.0)$, duodenum ( $\mathrm{pH} 5.5)$, jejunum $(\mathrm{pH}$ $6.0)$ and ileum ( $\mathrm{pH} 7.2 \sim 8.0)$. Therefore, they developed the vaccine carrier having $\mathrm{pH}$-sensitivity and selective delivery of vaccines into $\mathrm{M}$ cells in Peyer's patches located at neutral $\mathrm{pH}$ of the intestine as shown in Fig. 5 [52]. As results, the thiolated HPMCP (T-HPMCP) increased mucoadhesive property of T-HPMCP microparticles prepared by emulsion method in the small intestine of porcine as shown in Fig. 6 [52] due to the disulfide bonds formed between thiol groups of the T-HPMCP and cysteine-rich glycoproteins of the mucus layer [53]. Also, M cell-homing peptide conjugated $\mathrm{BmpB}$ (outer membrane lipoprotein of the pathogenic intestinal spirochaete Brachyspira hydodysenteriae) vaccines were mostly delivered in Peyer's patches in the ileum due to the higher uptake of the vaccines via M-cell as shown in Fig. 7 [52]. Furthermore, the T-HPMCP induced not only strong antibody-mediated immune responses produced but also memory $\mathrm{T}$ cells in the spleen as adaptive immunity after oral immunization compared to the HPMCP, a suggestion of T-HPMCP for ileum-specific delivery of vaccine via oral immunization.

They also prepared a mannan-decorated mucoadhesive vaccine delivery carrier to overcome the rapid mucociliary clearance in the respiratory mucosa by thiolation and to target the pathogen recognition receptors (PRRs) by mannan decoration of the surface of the T-HPMCP microparticles (M-THM) prepared by the double emulsion method [54]. They loaded ApxIIA vaccine into the mannan-decorated T-HPMCP microparticles (M-THM) to protect A. pleuropneumonia known to cause contagious porcine pleuropneumonia [55]. The results indicated that the M-THM enhanced receptor-mediated endocytosis by stimulating the mannose receptors (MRs) of APCs as shown in Fig. 8 [54]. Also, the ApxIIA-loaded M-THM showed higher levels of mucosal sJgA and serum IgG than ApxIIA of ApxIIA-loaded THM groups owing to the specific recognition of the mannose in the M-THM by the MRs of the APCs after intranasal immunization in mice as shown in Fig. 9 [54]. Furthermore, ApxIIA-loaded M-THM protected immunized mice after challenged with strains of A. pleuropneumonia serotype 5 as shown in Fig. 10 [54], a suggestion of a promising carrier for the nasal vaccine delivery system for eliciting mucosal and 


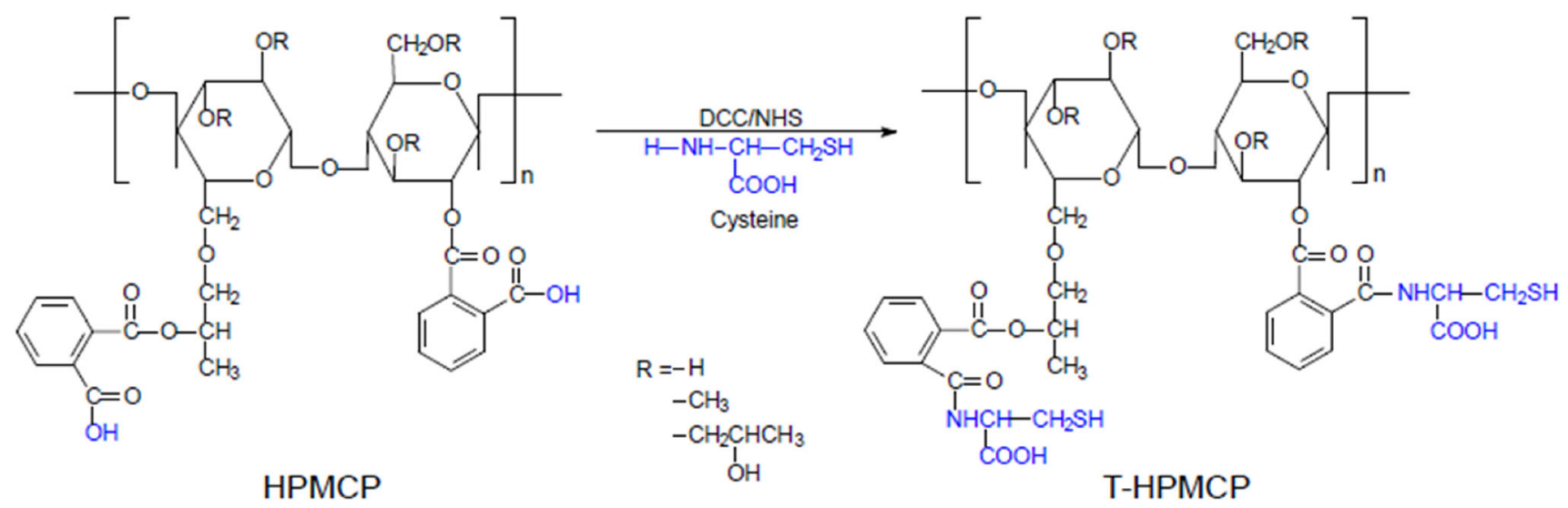

Fig. 4 The reaction scheme for the synthesis of T-HPMCP. Adapted from Singh et al., Attuning hydroxypropyl methylcellulose phthalate to oral delivery vehicle for effective and selective delivery of protein

vaccine in ileum. Biomaterials 2015, 59, $144 \sim 159$ with permission of Elsevier [52]



Fig. 5 Design for oral delivery of vaccines targeted to $M$ cells in ileum. Intraluminal $\mathrm{pH}$ and GI transit time are indicated (distance not to scale). Microparticles (MPs) are expected to begin to dissolve in the ileum for uptake of released antigens through $\mathrm{M}$ cells. Adapted
Grabovac et al., Comparison of the mucoadhesive properties of various polymers. Adv Drug Deliv Rev 2005, 57, $1713 \sim 1723$ with permission of Elsevier [53] 




B



Fig. 6 Analysis of morphology and size of MPs. Morphology of the MPs was analyzed by SEM (scale bar: $2 \mathrm{~mm}$ ). FITC-labeled antigen/ MPs were observed by CLSM. A M-BmpB/THPMCP MPs and FITCM-BmpB/T-HPMCP MPs (inset); B M-BmpB/HPMCP MPs and FITC-M-BmpB/HPMCP MPs (inset). The particle-size distributions

systemic immunity to protect from pathogenic bacteria infection.

\subsection{Eudragit-based mucoadhesive particles}

Eudragit polymers obtained synthetically from the esters of acrylic and methacrylic acid have shown many potentials in the conventional, $\mathrm{pH}$-sensitive, and novel drug delivery systems for loading of several kinds of drugs including genes, proteins, hormones, vitamins, and vaccines due to the different grades of Eudragit polymers by changing functional groups. They can be used for the stomachspecific, colon-specific, and mucosal delivery. Also, they can be used in the formulation of particulate systems. Therefore, Eudragit series as $\mathrm{pH}$-sensitive copolymers of poly (methacrylic acid-co-methacrylate) have been widely used in medical and pharmaceutical applications. In this



D



were detected by DLS. C MBmpB/T-HPMCP MPs; D M-BmpB/ HPMCP MPs. Adapted from Singh et al., Attuning hydroxypropyl methylcellulose phthalate to oral delivery vehicle for effective and selective delivery of protein vaccine in ileum. Biomaterials 2015, 59, 144-159 with permission of Elsevier [53]

section, we discuss mucosal vaccine delivery using Eudragit-based mucoadhesive particles.

Cui et al. prepared bilayer films composed of a thin wax layer bonded to a mucoadhesive layer having a crosslinked polyacrylate and Eudragit S-100 to load plasmid DNA vaccine containing a cytomegalovirus (CMV) promoter and $\beta$-galactosidase to induce both cellular and humoral immune responses [56]. The results indicated that the weight ratio of cross-linked polyacrylate and Eudragit S-100 affected mucoadhesive property with the remaining stability of the released vaccines from bilayer films. Also, vaccine-loaded bilayer films after buccal immunization in rabbits showed comparable antigen-specific IgG titer compared to that of subcutaneous injection. Furthermore, all rabbits immunized with DNA vaccine-loaded bilayer films showed splenocyte proliferative immune responses but none by the subcutaneous immunization although they did not check the immune responses by the particle system. 


4Fig. 7 Localization of FITC-labeled M-BmpB in Peyer's patch of mouse small intestine. A FITC-labeled M-BmpB/T-HPMCP or M-BmpB/HPMCP MPs were orally administered into the mice and their localization was monitored under fluorescence-microscopy. The green fluorescent signals of FITC-labeled $\mathrm{M}-\mathrm{BmpB}$, when delivered by T-HPMCP MPs, were higher in Peyer's patch underneath the FAE region. B Uptake of FITC-M-BmpB was quantitated by image J analysis and normalized to a value of 1.0 for $\mathrm{M}-\mathrm{BmpB}$ control. Scale bar: $200 \mathrm{~mm}$. (For interpretation of the references to color in this figure legend, the reader is referred to the web version of this article.) Adapted from Singh et al., Attuning hydroxypropyl methylcellulose phthalate to oral delivery vehicle for effective and selective delivery of protein vaccine in ileum. Biomaterials 2015, 59, $144 \sim 159$ with permission of Elsevier [52]

Pastor et al. prepared mucoadhesive Eudragit L 30 D-55 microparticles with alginate to load vibrio cholera vaccine for prolonging residence time and providing a concentration gradient at the mucosa membrane of the vaccine [57]. The results indicated that gastro resistance and antigenicity values of cholera vaccine-loaded Eudragit alginate microparticles were kept in an acceptable range. Also, the Eudragit/alginate microparticles induced stronger immune responses compared to the free vibrio vaccine after oral immunization in rats due to the gastro-resistant and mucoadhesive properties of the Eudragit/alginate.

Singh et al. prepared $\mathrm{pH}$-sensitive and mucoadhesive thiolated Eudragit L-100 microparticles (TEM) by double emulsion method to load $\mathrm{BmpB}$ vaccine for protection of a contagious mucohaemorrhagic colitis of pigs [58]. The results indicated that about $22-23 \mathrm{wt} \%$ of $\mathrm{BmpB}$ were released from the BmpB-loaded TEM at $\mathrm{pH} 2.0$ within $24 \mathrm{~h}$ whereas the release of $\mathrm{BmpB}$ from the BmpB-loaded TEM was $81 \mathrm{wt} \%$ at $\mathrm{pH} 7.2$ within $24 \mathrm{~h}$ due to the $\mathrm{pH}-$ sensitive property of the TEM. Also, the TEM showed a higher binding affinity with the mucin glycoproteins of the porcine intestine than Eudragit microparticles (EM) [58] due to the mucoadhesive property of the TEM. Furthermore, TNF- $\alpha$ production from Raw 264.7 cells treated with BmpB-loaded TEM was higher than EM or lipopolysaccharide although they did not perform in vivo study. Also, they prepared mannan-decorated TEM (M-TEM) by double emulsion method for targeting APCs after loading of OVA as the model vaccine [59]. The results indicated that M-TEM showed receptor-mediated endocytosis by stimulating the mannose receptors of APCs. Also, OVA-loaded M-TEM enhanced higher levels of serum IgG and mucosal sIgA than OVA itself after nasal vaccination in mice due to the specific recognition of mannose receptors of APCs by the mannose groups in the mannan of the M-TEM, suggesting a promising candidate to elicit mucosal and systemic immunity.

\subsection{Hybrid-based mucoadhesive particles}

Biodegradable polymeric particles such as poly (lactic-coglycolic acid) (PLGA) particles have been extensively used for the delivery of proteins including vaccines. However, PLGA has limited use in mucosal immunization due to its poor mucoadhesion and rapid clearance time of about 20 min from the human nasal cavity [60] because such a rapid clearance does not provide sufficient retention of vaccine to be taken up by APCs in the nasal-associated lymphoid tissue (NALT) [61]. Therefore, incorporation of mucoadhesive polymers such as chitosan derivative-, cellulose derivative- and Eudragit-based polymers can overcome such limitations and enhance absorption of the vaccines across the mucosal barrier by prolonging their residence time in the mucosal cavity [62]. In this section, we discuss hybrid mucoadhesive particles for mucosal immunization.

Pawar et al. prepared TMC-coated PLGA microparticles by double emulsion method to load hepatitis B surface antigen (HBsAg) for protection of hepatitis B virus (HBV) infection as one of the most prevalent chronic viral infections worldwide [61]. The results indicated that TMCcoated PLGA microparticles showed higher mucin adsorption than chitosan-coated PLGA ones or plasm PLGA ones due to the mucoadhesive property of the TMC. Also, HBsAg-loaded TMC-coated PLGA microparticles showed higher anti-HBsAg titer in serum and secretions compared to HBsAg-loaded chitosan-coated PLGA ones after nasal immunization in mice. They also prepared GCcoated PLGA nanoparticles by double emulsion method to load HBsAg for protecting HBV infection [63]. The results indicated that GC-coated PLGA nanoparticles showed lower clearance and better uptake of antigens compared to chitosan-coated PLGA nanoparticles or uncoated PLGA ones due to the mucoadhesive property of the GC. Also, GC-coated PLGA nanoparticles induced significantly higher mucosal and systemic immune responses in serum and secretory than chitosan-coated PLGA nanoparticles or PLGA ones after nasal immunization in mice, an indication of a promising nasal vaccine delivery carrier for inducing a potent immune response at mucosal sites and systemic circulation.

Rose et al. prepared GC-coated lipid/PLGA hybrid nanoparticles (GC-LPNs) by a single emulsion method to load recombinant chlamydia trachomatis fusion antigen CTH522 for protection of chlamydia trachomatis $(\mathrm{Ct})$ as the most common sexually transmitted infection in the world [64]. The results indicated that a PLGA core was coated with lipid bilayers and the GC coating of the LPNs was identified as saturable with a GC concentration-dependent increase of nanoparticle size and a reduction of the zeta-potential. Also, increased CTH522-specific IgG/IgA 



\section{B}

\section{Raw264.7 uptaking assay}



Fig. 8 A Confocal microscopic images of RAW264.7 cells after $2 \mathrm{~h}$ culture with OVA-loaded THMand OVA-loaded Man-THMat $4{ }^{\circ} \mathrm{C}$ and $37{ }^{\circ} \mathrm{C}$. B Measurement of microsphere uptake by RAW264.7 using FACS. Uptake of OVA-FITC-loaded THM and OVA-FITC loaded Man-THM by RAW 264.7 in $1 \mathrm{~h}$ and $2 \mathrm{~h}$ with and without MR inhibition at $37{ }^{\circ} \mathrm{C}(\mathrm{n}=3$, error bar represents standard

deviation; $* p<0.05, * * p<0.01, * * * p<0.005$, one-way ANOVA). Adapted from Li et al., Nasal immunization with mannan-decorated mucoadhesive HPMCP microspheres containing ApxIIA toxin induces protective immunity against challenge infection with Actinobacillus pleuropneumoiae in mice. Journal of Controlled Release 2016, 233, 114-125 with permission of Elsevier [54]

antibodies with CTH522-specific interferon $\gamma$-producing Th1 cells were more induced with the GC-LPNs after nasal immunization in mice than LPNs or CTH522 antigen due to the mucoadhesive property of the GC, suggesting a

promising strategy to enhance the mucosal vaccine responses.

Quan et al. prepared thiolated Eudragit-coated chitosan microspheres (TECMs) by ionic gelation method to load bovine serum albumin (BSA) as an antigen model drug 
Fig. 9 A-C ApxIIA-specific IgA performance in themucosal sites at 4 weeks postimmunization. ApxIIA-specific brochealveolar lavage (A), nasal wash $(\mathbf{B})$, vaginal wash $(\mathbf{C}), \operatorname{Ig}$ A levels inmice immunizedwith the indicated formulationswere analyzed by ELISA and then calculated by optical density $(450 \mathrm{~nm})(\mathrm{n}=5$, error bars represent standard deviations;

$* p<0.05, * * p<0.01$,

$* * * p<0.005$, one-way

ANOVA). Adapted from Li

et al., Nasal immunization with mannan-decorated mucoadhesive HPMCP

microspheres containing ApxIIA toxin induces protective immunity against challenge infection with Actinobacillus pleuropneumoiae in mice. Journal of Controlled Release 2016, 233, 114-125 with permission of Elsevier [54]
A


B
C

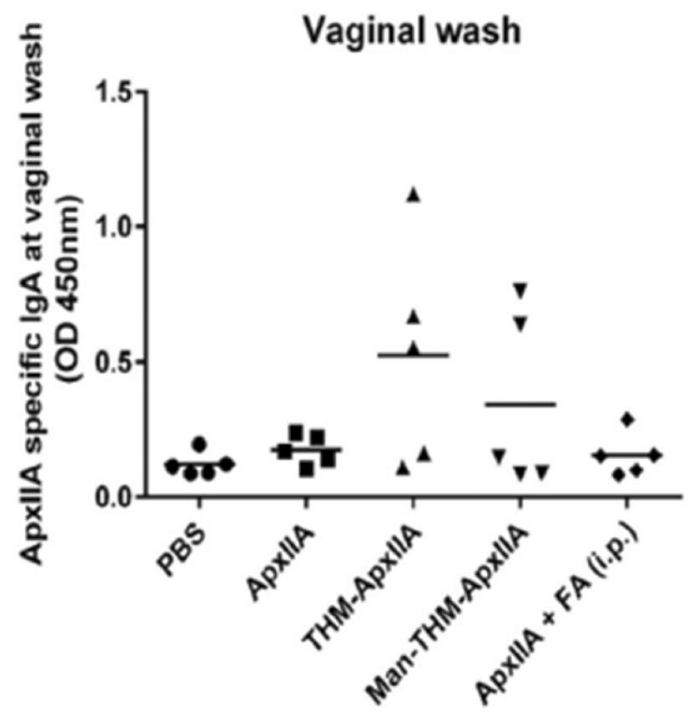

[65]. The results indicated that the release of BSA from BSA-loaded TECMs was suppressed at $\mathrm{pH} 2.0$ whereas the BSA from BSA-loaded TECMs was released sustainingly for several hours at $\mathrm{pH} 7.4$ due to the $\mathrm{pH}$-sensitivity of the thiolated Eudragit. Also, more TECMs remained on the isolated porcine intestinal mucosal surface than Eudragitcoated chitosan microspheres in vitro and Tc-99 m-labeled TECMs observed by gamma camera imaging were more distributed in the rat intestine after oral administration as shown in Fig. 11 [65] although they did not check immune responses.

Gupta et al. prepared liposome gel-based formulations containing HEC to load HIV-1 envelope glycoprotein, CN54gp140 for protection of HIV infection in the vaginal 
A



B



Fig. 10 Induction of protective immunity after intranasal challenge with A. pleuropneumoniae. A 14 days after the last immunization, 5 mice per group were challenged intranasally with a minimal lethal dose $(5 \times 107 \mathrm{CFU})$ of $\mathrm{A}$. pleuropneumoniae,with the survival rate (\%)monitored for an additional 4 days. B The number of residual bacteria was counted per $100 \mathrm{mgfresh}$ lung tissue weight from each mice per group. $(p<0.05, * * p<0.01, * * * p<0.005$, one-way



= THM-ADXIIA

III Man-THM-ApxuIA

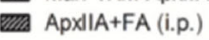

C
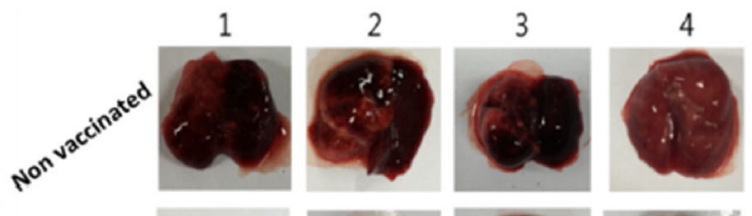

5


ANOVA) C The lungs were characterized before becoming homogenates and after bacteria challenge. Adapted from Li et al., Nasal immunization with mannan-decorated mucoadhesive HPMCP microspheres containing ApxIIA toxin induces protective immunity against challenge infection with Actinobacillus pleuropneumoiae in mice. Journal of Controlled Release 2016, 233, 114-125 with permission of Elsevier [54]
Fig. 11 Amount of FDA remained on excised porcine small intestinal mucosa.

Adapted from Quan et al., pHsensitive and mucoadhesive thiolated Eudragit-coated chitosan microspheres. International Journal of Pharmaceutics 2008, $359,205-210$ with permission of Elsevier [65]




[66]. The results indicated that chitosan and HPMC weight ratio of $8 / 2$ showed controlled release of TT vaccine by 90 days in vitro after stabilization of the vaccine with heparin. Also, TT-loaded chitosan/HPMC showed a higher antibody level $(4.5 \mathrm{IU} / \mathrm{ml})$ than that of alum-adsorbed $\mathrm{TT}(2 \mathrm{IU} / \mathrm{ml})$ in serum after intraperitoneal immunization in a guinea pig, an indication of a promising single-step immunization with vaccines although they did not check mucosal immunization.

\section{Conclusion and future perspectives}

The mucosal immunization may result in the induction of protective immune responses in organized lymphoid tissues at mucosal sites. However, enzymatic degradation and fast mucociliary clearance of antigens in the mucosal sites decrease the bioavailability of antigens and limit to a generation of adequate mucosal immune responses against used vaccines. Therefore, efficient vaccine delivery systems should be used. Among them, mucosally particulate vaccine delivery systems are one of the alternative ways because they can protect enzymatic degradation, can target mucosal inductive sites, and can control the release of loaded vaccines. Especially, mucoadhesive polymeric particles can additionally prolong the residence time of the used vaccines at the target site.

In this review, we covered chitosan derivative-based, cellulose derivative-based, Eudragit-based, and hybridbased mucoadhesive polymeric particles for mucosal vaccination.

Unmodified chitosan has limitations of insolubility at neutral $\mathrm{pH}$ and rapid clearance from the body although it has biocompatibility, biodegradability, and low toxicity. Recently many researchers have been studied to develop the second-generation mucoadhesive chitosan derivativesbased mucosal vaccine delivery carriers such as thiolated chitosan, TMC, and quaternized chitosan derivatives. However, the research of chitosan derivatives-based vaccine delivery carriers is still in the preclinical stage because the chitosan is a mixture, its degree of deacetylation, its molecular weight, and different degree of quaternization of chitosan, which affects great differences in immune responses. Therefore, physicochemical properties of the mucoadhesive chitosan derivatives-based mucosal vaccine delivery carriers should be optimized for clinical applications. Also, a new strategy for the targeted vaccine delivery using various specific ligands such as mannose, folate, galactose, and $\mathrm{M}$ cell targeting will be very promising.

Cellulose derivative-based and Eudragit-based thiolated mucoadhesive particles may be expected to use for vaccine delivery in clinical trials because HPMCP and Eudragit have been approved in pharmaceutical applications by
FDA. However, the size, shape, and change of polymeric particles should be taken into account for effective mucosal vaccine delivery because they determine the endocytosis pathway of polymeric particles into APCs that subsequently affect the way of presentation of antigens to the immune cells [11]. Furthermore, the mechanism at molecular levels and cooperation among researchers in immunology, materials science, and bioengineering should be needed for enhancing immune responses. The authors hope that this review will provide useful stimuli for encouraging future research and development of efficient mucosal vaccine delivery systems.

Acknowledgements This research was supported by Basic Science Research Program through the National Research Foundation of Korea(NRF) funded by the Ministry of Education (NRF2020R1I1A1A01053275). Also, this research was supported by Republic of Korea, and Korea Institute of Planning and Evaluation for Technology in Food, Agriculture and Forestry (IPET) through Animal Disease Management Technology Development Program, funded by Ministry of Agriculture, Food and Rural Affairs (MAFRA) (31908103).

\section{Compliance with ethical standards}

Conflicts of interest The authors have no financial conflicts of interest.

Ethical statement There are no animal experiments carried out for this article.

\section{References}

1. Corthésy B, Bioley G. Lipid-based particles: versatile delivery systems for mucosal vaccination against infection. Front Immunol. 2018;9:431.

2. Yoon SY, Kang SK, Lee HB, Oh SH, Kim WS, Li HS, et al. Enhanced efficacy of immunization with a foot-and-mouth disease multi-epitope subunit vaccine using mannan-decorated inulin microparticles. Tissue Eng Regen Med. 2020;17:33-44.

3. Lim W, Kim HS. Exosomes as therapeutic vehicles for cancer. Tissue Eng Regen Med. 2019;16:213-23.

4. Pulendran B, Ahmed R. Immunological mechanisms of vaccination. Nat Immunol. 2011;12:509-17.

5. Longet S, Lundahl MLE, Lavelle EC. Targeted strategies for mucosal vaccination. Bioconjug Chem. 2018;29:613-23.

6. Lycke N. Recent progress in mucosal vaccine development: potential and limitations. Nat Rev Immunol. 2012;12:592-605.

7. Woodrow KA, Bennett KM, Lo DD. Mucosal vaccine design and delivery. Annu Rev Biomed Eng. 2012;14:17-46.

8. Nguyen L, Bang S, Noh I. Tissue regeneration of human mesenchymal stem cells on porous gelatin micro-carriers by longterm dynamic in vitro culture. Tissue Eng Regen Med. 2019;16:19-28.

9. Kim TW, Ahn WB, Kim JM, Kim JH, Kim TH, Perez RA, et al. Combined delivery of two different bioactive factors incorporated in hydroxyapatite microcarrier for bone regeneration. Tissue Eng Regen Med. 2020;17:607-24.

10. Czerkinsky C, Anjuere F, McGhee JR, George-Chandy A, Holmgren J, Kieny MP, et al. Mucosal immunity and tolerance: 
relevance to vaccine development. Immunol Rev. 1999; 170:197-222.

11. Singh B, Maharjan S, Cho KH, Cui L, Park IK, Choi YJ, et al. Chitosan-based particulate systems for the delivery of mucosal vaccines against infectious diseases. Int $\mathrm{J}$ Biol Macromol. 2018;110:54-64.

12. Andrianov AK, Payne LG. Polymeric carriers for oral uptake of microparticulates. Adv Drug Deliv Rev. 1998;34:155-70.

13. Amidi M, Mastrobattista E, Jiskootv W, Hennink WE. Chitosanbased delivery systems for protein therapeutics and antigens. Adv Drug Deliv Rev. 2010;62:59-82.

14. Ko KW, Yoo YJ, Kim JY, Choi B, Park SB, Park W, et al. Attenuation of tumor necrosis factor- $\alpha$ induced inflammation by umbilical cord-mesenchymal stem cell derived exosome-mimetic nanovesicles in endothelial cells. Tissue Eng Regen Med. 2020;17:155-63.

15. Goyal AK, Singh R, Chauhan G, Rath G. Non-invasive systemic drug delivery through mucosal routes. Artif Cells Nanomed Biotechnol. 2018;46:539-51.

16. Dewangan HK. Rational application of nanoadjuvant for mucosal vaccine delivery system. J Immunol Methods. 2020;481-2:112791.

17. Bahadoran A, Moeini H, Bejo MH, Hussein MZ, Omar AR. Development of tat-conjugated dendrimer for transdermal DNA vaccine delivery. J Pharm Pharm Sci. 2016;19:325-38.

18. Bhavsar MD, Aniji MM. Polymeric nano and micro particle technologies for oral gene delivery. Expert Opin Drug Deliv. 2007;4:197-213.

19. Wallis J, Shenton DP, Carlisle RC. Novel approaches for the design, delivery and administration of vaccine technologies. Clin Exp Immunol. 2019;196:189-204.

20. Lehtinen M, Luostarinen $T$, Vänskä S, Söderlund-Strand $S$, Eriksson T, Natunen $\mathrm{K}$, et al. Gender-neutral vaccination provides improved control of human papillomavirus types 18/31/33/ 35 through herd immunity: results of a community randomized trial (III). Int J Cancer. 2018;143:2299-310.

21. Lycke N. Recent progress in mucosal vaccine development: potential and limitations. Nat Rev Immunol. 2012;12:592-605.

22. Shakya AK, Chowdhury MY, Tao W, Gill HS. Mucosal vaccine delivery: current state and a pediatric perspective. J Control Release. 2016;240:394-413.

23. Kozlowski PA, Williams SB, Lynch RM, Flanigan TP, Patterson $\mathrm{RR}, \mathrm{Cu}-\mathrm{Uvin} \mathrm{S}$, et al. Differential induction of mucosal and systemic antibody responses in women after nasal, rectal, or vaginal immunization: influence of the menstrual cycle. J Immunol. 2002;169:566-74.

24. Lehtinen M, Söderlund-Strand A, Vänskä S, Luostarinen T, Eriksson T, Natunen K, et al. Impact of gender-neutral or girlsonly vaccination against human papillomavirus-results of a community-randomized clinical trial (I). Int $\mathrm{J}$ Cancer. 2018;142:949-58.

25. Nesburn AB, Bettahi I, Zhang X, Zhu X, Chamberlain W, Afifi $\mathrm{RE}$, et al. Topical/mucosal delivery of sub-unit vaccines that stimulate the ocular mucosal immune system. Ocul Surf. 2006;4:178-87.

26. Moffatt K, Wang Y, Singh TR, Donnelly RF. Microneedles for enhanced transdermal and intraocular drug delivery. Curr Opin Pharmacol. 2017;36:14-21.

27. Vasisht N, Gever LN, Tagarro I, Finn AL. Single-dose pharmacokinetics of fentanyl buccal soluble film. Pain Med. 2010;11:1017-23.

28. Ahmed TA, Aljaeid BM. Preparation, characterization, and potential application of chitosan, chitosan derivatives, and chitosan metal nanoparticles in pharmaceutical drug delivery. Drug Des Devel Ther. 2016;10:483-507.
29. Calderón L, Harris R, Cordoba-Diaz M, Elorza M, Elorza B, Lenoir J, et al. Nano and microparticulate chitosan-based systems for antiviral topical delivery. Eur J Pharm Sci. 2013;48:216-22.

30. Islam MA, Park TE, Reesor E, Cherukula K, Hasan A, Firdous J, et al. Mucoadhesive Chitosan Derivatives as Novel Drug Carriers. Curr Pharm Des. 2015;21:4285-309.

31. Brown MA, Daya MR, Worley JA. Experience with chitosan dressings in a civilian EMS system. J Emerg Med. 2009;37:1-7.

32. Xin-Yuan S, Tian-Wei T. New contact lens based on chitosan/ gelatin composites. J Bioact Compat Polym. 2004;19:467-79.

33. Meng J, Zhang T, Agrahari V, Ezoulin MJ, Youan BB. Comparative biophysical properties of tenofovir-loaded, thiolated and nonthiolated chitosan nanoparticles intended for HIV revention. Nanomedicine (Lond). 2014;9:1595-612.

34. Richardson SC, Kolbe HV, Duncan R. Potential of low molecular mass chitosan as a DNA delivery system: biocompatibility, body distribution and ability to complex and protect DNA. Int J Pharm. 1999;178:231-43.

35. Kast CE, Bernkop-Schnürch A. Thiolated polymers-thiomers: development and in vitro evaluation of chitosan-thioglycolic acid conjugates. Biomaterials. 2001;22:2345-52.

36. Verheul RJ, Slütter B, Bal SM, Bouwstra JA, Jiskoot W, Hennink WE. Covalently stabilized trimethyl chitosan-hyaluronic acid nanoparticles for nasal and intradermal vaccination. J Control Release. 2011;15:46-52.

37. Sinani G, Sessevmez M, Gök MK, Özgümüş S, Alpar HO, Cevher E. Modified chitosan-based nanoadjuvants enhance immunogenicity of protein antigens after mucosal vaccination. Int J Pharm. 2019;569:118592.

38. Cevher E, Taha MA, Orlu M, Araman A. Evaluation of mechanical and mucoadhesive properties of clomiphene citrate gel formulations containing carbomers and their thiolated derivatives. Drug Deliv. 2008;15:57-67.

39. Bhavsar C, Momin M, Gharat S, Omri A. Functionalized and graft copolymers of chitosan and its pharmaceutical applications. Expert Opin Drug Deliv. 2017;14:1189-204.

40. Marasini N, Giddam AK, Khalil ZG, Hussein WM, Capon RJ, Batzloff MR, et al. Double adjuvanting strategy for peptidebased vaccines: trimethyl chitosan nanoparticles for lipopeptide delivery. Nanomedicine (Lond). 2016;11:3223-35.

41. Li H, Lu Y, Xiang J, Jiang H, Zhong Y, Lu Y. Enhancement of immunogenic response and protection in model rats by CSTM nanoparticles anticaries DNA vaccine. Nanomedicine (Lond). 2016;11:1407-16.

42. Abkar M, Fasihi-Ramandi M, Kooshki H, Sahebghadam Lotfi A. Oral immunization of mice with Omp31-loaded N-trimethyl chitosan nanoparticles induces high protection against brucella melitensis infection. Int J Nanomedicine. 2017;12:8769-78.

43. Nevagi RJ, Khalil ZG, Hussein WM, Powell J, Batzloff MR, Capon RJ, et al. Polyglutamic acid-trimethyl chitosan-based intranasal peptide nanovaccine induces potent immune responses against group A streptococcus. Acta Biomater. 2018;80:278-87.

44. Nevagi RJ, Dai W, Khalil ZG, Hussein WM, Capon RJ, Skwarczynski $M$, et al. Self-assembly of trimethyl chitosan and poly(anionic amino acid)-peptide antigen conjugate to produce a potent self-adjuvanting nanovaccine delivery system. Bioorg Med Chem. 2019;27:3082-8.

45. Jearanaiwitayakul T, Sunintaboon $P$, Chawengkittikul R, Limthongkul J, Midoeng P, Warit S, et al. Nanodelivery system enhances the immunogenicity of dengue-2 nonstructural protein 1, DENV-2 NS1. Vaccine. 2020;38:6814-25.

46. Zhao K, Sun Y, Chen G, Rong G, Kang H, Jin Z, et al. Biological evaluation of N-2-hydroxypropyl trimethyl ammoniumchloride chitosan as a carrier for the delivery of live Newcastle diseasevaccine. Carbohydr Polym. 2016;149:28-39. 
47. Zhao K, Li S, Li W, Yu L, Duan X, Han J, et al. Quaternized chitosan nanoparticles loaded with the combined attenuated live vaccine against newcastle disease and infectious bronchitis elicit immune response in chicken after intranasal administration. Drug Deliv. 2017;24:1574-86.

48. Zhao K, Han J, Zhang Y, Wei L, Yu S, Wang X, et al. Enhancing mucosal immune response of newcastle disease virus DNA vaccine using N-2-hydroxypropyl trimethylammonium chloride chitosan and $\mathrm{N}$, O-carboxymethyl chitosan nanoparticles as delivery carrier. Mol Pharm. 2018;15:226-37.

49. Gogev S, de Fays K, Versali MF, Gautier S, Thiry E. Glycol chitosan improves the efficacy of intranasally administrated replication defective human adenovirus type 5 expressing glycoprotein D of bovine herpesvirus 1. Vaccine. 2004;22:1946-53.

50. Pawar D, Jaganathan KS. Mucoadhesive glycol chitosan nanoparticles for intranasal delivery of hepatitis B vaccine: enhancement of mucosal and systemic immune response. Drug Deliv. 2016;23:185-94.

51. Donnelly L, Curran RM, Tregoning JS, McKay PF, Cole T, Morrow RJ, et al. Intravaginal immunization using the recombinant HIV-1 clade-C trimeric envelope glycoprotein CN54gp140 formulated within lyophilized solid dosage forms. Vaccine. 2011;29:4512-20.

52. Singh B, Maharjan S, Jiang T, Kang SK, Choi YJ, Cho CS. Attuning hydroxypropyl methylcellulose phthalate to oral delivery vehicle for effective and selective delivery of protein vaccine in ileum. Biomaterials. 2015;59:144-59.

53. Grabovac V, Guggi D, Bernkop-Schnürch A. Comparison of the mucoadhesive properties of various polymers. Adv Drug Deliv Rev. 2005;57:1713-23.

54. Li HS, Shin MK, Singh B, Maharjan S, Park TE, Kang SK, et al. Nasal immunization with mannan-decorated mucoadhesive HPMCP microspheres containing ApxIIA toxin induces protective immunity against challenge infection with actinobacillus pleuropneumoiae in mice. J Control Release. 2016;233:114-25.

55. Bento D, Staats HF, Gonçalves T, Borges O. Development of a novel adjuvanted nasal vaccine: $\mathrm{C} 48 / 80$ associated with chitosan nanoparticles as a path to enhance mucosal immunity. Eur J Pharm Biopharm. 2015;93:149-64.

56. Borde A, Ekman A, Holmgren J, Larsson A. Effect of protein release rates from tablet formulations on the immune response after sublingual immunization. Eur $\mathrm{J}$ Pharm Sci. 2012;47:695-700.
57. Gupta PN, Pattani A, Curran RM, Kett VL, Andrews GP, Morrow $\mathrm{RJ}$, et al. Development of liposome gel based formulations for intravaginal delivery of the recombinant HIV-1 envelope protein CN54gp140. Eur J Pharm Sci. 2012;46:315-22.

58. Ozbılgın ND, Saka OM, Bozkır A. Preparation and in vitro/ in vivo evaluation of mucosal adjuvant in situ forming gels with diphtheria toxoid. Drug Deliv. 2014;21:140-7.

59. Li HS, Singh B, Park TE, Hong ZS, Kang SK, Cho CS, et al. Mannan-decorated thiolated eudragitmicrospheres for targeting antigen presenting cells via nasal vaccination. Eur J Pharm Sci. 2015;80:16-25.

60. Illum L. Nasal drug delivery-possibilities, problems and solutions. J Control Release. 2003;87:187-98.

61. Pawar D, Goyal AK, Mangal S, Mishra N, Vaidya B, Tiwari S, et al. Evaluation of mucoadhesive PLGA microparticles for nasal immunization. AAPS J. 2010;12:130-7.

62. Soane RJ, Hinchcliffe M, Davis SS, Illum L. Clearance characteristics of chitosan based formulation in the sheep nasal cavity. Int J Pharm. 2001;217:183-91.

63. Pawar D, Mangal S, Goswami R, Jaganathan KS. Development and characterization of surface modified PLGA nanoparticles for nasal vaccine delivery: effect of mucoadhesive coating on antigen uptake and immune adjuvant activity. Eur J Pharm Biopharm. 2013;85:550-9.

64. Rose F, Wern JE, Gavins F, Andersen P, Follmann F, Foged C. A strong adjuvant based on glycol-chitosan-coated lipid-polymer hybrid nanoparticles potentiates mucosal immune responses against the recombinant chlamydia trachomatis fusion antigen CTH522. J Control Release. 2018;271:88-97.

65. Quan JS, Jiang HL, Kim EM, Jeong HJ, Choi YJ, Guo DD, et al. $\mathrm{pH}$-sensitive and mucoadhesive thiolated eudragit-coated chitosan microspheres. Int J Pharm. 2008;359:205-10.

66. Arthanari S, Mani G, Peng MM, Jang HT. Chitosan-HPMCblended microspheres as a vaccine carrier for the delivery of tetanus toxoid. Artif Cells Nanomed Biotechnol. 2016;44:517-23.

Publisher's Note Springer Nature remains neutral with regard to jurisdictional claims in published maps and institutional affiliations. 